\title{
Third and Fourth Thoughts About Research Instrumentation
}

\author{
Rustum Roy \\ Pennsylvania State University
}

\begin{abstract}
$T^{3}$ he Council of the National Academies of Science and Engineering has recently reminded the community of the dangers inherent in certain developments in the seeking of Federal funding for research and instrumentaton. Its statement of October 30, 1983 says:

"We urge that the academic community and public officials exercise vigilance to protect this informed evaluation and decisionmaking process in the awarding of funds, not only for the support of scientific research proposals, but also for major scientific facilities and instrumentation."
\end{abstract}

The Academy was reacting to extraordinary actions beginning with the attempt of George A. Keyworth, President Reagan's Science Advisor, to allocate very large sums of money (some $\$ 200$ million over a few years) to the University of California's Lawrence Berkeley Laboratory for a synchrotron light source, under the label of "materials research." This was blocked by an aroused materials community writing letters to their Congressional representatives, pointing out that no materials group had ever said materials research saw synchrotrons as the highest priority item. But this attempt started a forest fire of other Congressional representatives' efforts to obtain direct funding for pet projects in their own districts: Catholic University (for a Vitreous State Laboratory), Columbia University (for a chemistry facility), University of Florida (for a supercomputer), and on and on.

There is no way we can say with certainty that their actions are bad or good for "science" or the "universityworld" or whatever; but it is certainly different from the way the Federal government has funded science these last 40 years. These efforts represent dissatisfaction within and outside the science community about resource allocations in science. How much money should we devote to radioastronomy as compared to materials research or biotechnology? And some are asking, if Navy shipyards can be allocated by the political process why not supercomputers for materials research?

These situations led to second thoughts on the part of some scientists about how to get what they wanted. They were largely physicists interested in high-intensity light and neutron sources which require big expensive machines. A committee was very properly established under the auspices of the NAS with an extraordinarily narrow charge: to come up with a list of big ( $>5$ or 10 million dollar) machines which were "needed" by the "materials community." This committee, the SeitzEastman Committee, has very conscientiously done its work (666 pages in the Appendix alone) and reported a list of instruments which it believes shows the priority needs, if big machines are indeed "needed." The price tag for building and operating some half-dozen facilities is nearly a billion dollars over several years.

As a materials scientist who is also a science policy analyst, I lean back in my armchair, reflect on these facts, and say: wait a minute, I have some third and fourth thoughts. The third thoughts have occurred to a very large number of other materials researchers including their senior research administrators in Washington (see R. A. Reynolds, Science, 226, p. 494). They occurred to many on the Seitz-Eastman Committee, and led them to put in various demurrer clauses in their report. They are very simple thoughts: Who speaks for materials research? (Why not indeed, the Materials Research Society the only society wholly devoted to that field?)

The Seitz-Eastman Committee-as they properly acknowledge - did not speak for all of materials research The charge to the Committee defined the outcome by limiting it to "big instruments," when the total materials research community would likely choose to put such items way down on a priority list ranked by costeffectiveness for today's national needs. But just "being against" another super TEM or supercomputer of 6.5 $\mathrm{GeV}$ synchrotron is like being against motherhood. Even beginning students in science policy class learn never to compare something against nothing. Let's phrase the question the only way a national policy maker can responsibly phrase it. Given the present state of U.S. materials science and U.S. technology, if you were to invest an extra $\$ 100$ to $\$ 200$ million of public funds per year for the next five years for the benefit of the $U$. S. materials research capability, where would you put the money?

Option A: A synchrotron or two plus some pulsed neutron sources.

Option B: $20-30$ centers/facilities devoted to a spectrum of major focussed projects including at least one each in the following areas: polymer systems and processing; ceramic finishing; GaAs growth; specialty single-crystal growth; welding and joining research; ceramic processing; electromagnetic nanocomposites; structural composites (brittle matrix and compliant matrix); new low-temperature energy conserving materials; corrosion inhibition; thin films, plasma sprays/laser coatings; etc.

Option C: Some mix of A and B. What mix and what order of priority?

High administration officials have urged the science community to set its priorities. But while "materials research" is being sold to the public as a panacea which it is not, no comprehensive examination of priorities-like a COSMAT II-has been started. Yet discipline-driven studies by the Academy in physics and chemistry of materials have been reported at the same time, with various inferences to the physics and chemistry of materials which could confuse policy makers. It is certain that in the very tight budgets that we will see that setting priorities which will really benefit the nation is an urgent task. The great danger confronting the materials 
research community of which our Society is the most comprehensive representative, is that one subgroup within the community will appropriate the name "materials research" - which is very fashionable today and speak on its behalf.

Nearly 15 years ago the first National Colloquy on the Field of Materials was held to discuss just such questions. A map of the field of materials research (Figure 1) reminds us of the nature of our field. Materials research includes many disciplines-but each of them is only a part of the whole. And only when they work interactively with the other disciplines do we call it materials research. Otherwise they are-obviously-doing physics, or electrical engineering, or geochemistry. My third thoughts end on the activist note that this Society and individual materials researchers who do not agree, should again express their opinions to their Congressional representatives and the right Congressional committees, for now attempts will certainly be made to claim that huge instrumental facilities are our most pressing need for materials research.

In closing: my fourth thought on this matter. How important is the super-sophisticated instrumentation anyway for the progress of U.S. materials science and especially technology? Are my present PhD students going to be more creative, more productive, more innovative than my first students 30 years ago because they can go from room to room down the hall doing surface analyses with $2 \square$ resolution, automated SEM with $50 \square$ resolution, ESCA, MASNMR, etc.? The answer is an emphatic no. While of course, these tools give them valuable new capabilities to push the limits of their problem, these tools do nothing for clear thinking or creativity. Indeed, I find that, in general, they tend to inhibit it, especially for technologically relevant science. There are so many instrument "trees" that the student more easily loses sight of the "forest" of the problem.

My own absolutely serious proposal for the most important need to advance American materials research is for Option $D$ to be added to the list above:

Option D: Purchase 20\% (one day/week) of the time of 5,000 of all the senior-most materials researchers in the country, and allocate it to doing absolutely nothing - just thinking about the problem, asking the deeper questions, questioning the usual approaches (including which instruments not to use), maybe even dreaming a little. (For 5,000 persons this would be only $\$ 50 \mathrm{M} /$ year (less including the negative overhead saving!) I feel certain this would provide more genuine technological innovation and more good science, than any alternative. Most good labs in the U.S don't lack instruments, they lack time to think.

The United States confronts annual national deficits of $\$ 200$ billion and foreign trade deficits of $\$ 120$ billion per year. I believe that scientists as citizens with special training must read the relevant literature and enquire as to the role of science and technology in alleviating these problems. My own analysis, developed in my book Lost at the Frontier (ISI Press, 1985) co-authored with Deborah Shapley, is that the United States does not have a balance between undirected basic research, long-range equally basic research relevant to technology, and engineering research. In undirected basic research we use up personnel in short supply and spend enormous sums, and we get what we pay for: more papers, more citations, more Nobel prizes (not as many per capita as the British). It is time to alter the balance towards applied or relevant science. The major step function advances in materials research have all been critically dependent on a new material or new process (nylon, pure germanium, pyroceram, synthetic diamonds, zerography, etc.) and as surely as the steam engine led to thermodynamics, each of these led to entire new branches of materials research Thus we see that decisions on large instruments versus technologically relevant centers are no longer merely a matter of research emphasis but are relevant to the health of the nation's economy. $M|\mathbf{R}| \mathbf{S}$

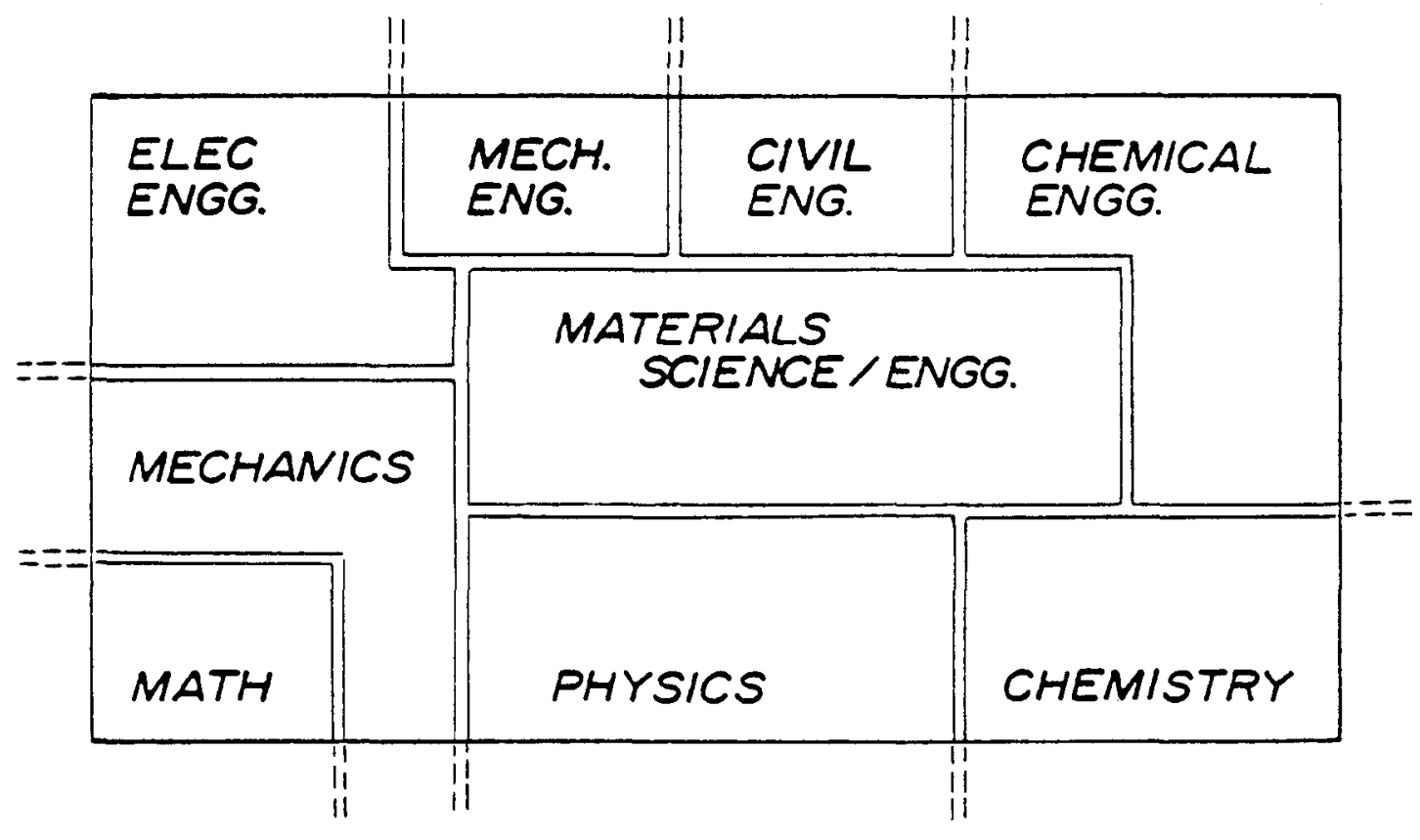

Figure 1

The scope of materials research. Note that scientists and engineers form a variety of disciplines all involved in research on materials. However in all these cases, materials research forms only a part of the research activity of the particular discipline: the single exception is the discipline of materials science/engineering. In that case alone does all the research of a practitioner lie wholly within the scope of materials research. [From Materials Science and Engineering in the U.S., p. 116. Edited by R. Roy., PSU Press, 1969.] 